\title{
La gouvernance territoriale contemporaine du Nitaskinan
}

\author{
Tradition, adaptation et flexibilité
}

The Contemporary Territorial Governance of Nitaskinan

Tradition, adaptation, and Flexibility

\section{La gobernanza territorial contemporánea de Nitaskinan}

Tradición, adaptación y flexibilidad.

\section{Otiperitamowin askik itekera Nitaskinan e arimotamotcikatek Otirosowin acitc e ici pimatisiwaketc irikik e pa icatc notcimik}

\section{Nicolas Houde}

Volume 44, numéro 1, 2014

Les Atikamekw Nehirowisiwok : territorialités et savoirs

URI : https://id.erudit.org/iderudit/1027877ar

DOI : https://doi.org/10.7202/1027877ar

Aller au sommaire du numéro

Éditeur(s)

Recherches amérindiennes au Québec

ISSN

0318-4137 (imprimé)

1923-5151 (numérique)

Découvrir la revue

Citer cet article

Houde, N. (2014). La gouvernance territoriale contemporaine du Nitaskinan : tradition, adaptation et flexibilité. Recherches amérindiennes au Québec, 44(1), 23-33. https://doi.org/10.7202/1027877ar
Résumé de l'article

La Nation atikamekw (Québec, Canada) est présentement engagée dans des négociations avec les gouvernements fédéral et provincial dans le but à la fois d'accroître son accès aux ressources naturelles et de transformer le système de gouvernance territoriale sur le Nitaskinan, le territoire ancestral nehirowisiw. Un enjeu central de cette redéfinition de la gouvernance est la revalorisation du rôle traditionnel et central du chef de territoire ( $k a$ nikaniwitc) dans ses décisions. Cet article montre que les Atikamekw Nehirowisiwok mettent présentement sur pied un système de gouvernance auquel ils s'identifient, ancré dans leurs traditions, centré sur le ka nikaniwitc et la famille, mais en même temps tourné vers l'avenir, évolutif et différent de sa forme originale. Dans ce système, le ka nikaniwitc se positionne comme un catalyseur par lequel passe et s'organise l'information concernant le territoire, afin de produire des décisions compatibles avec les valeurs nehirowisiwok et ayant des effets tant localement que régionalement. À la lumière des résultats présentés dans l'article, il est possible d'affirmer que, pour que la transformation de la gouvernance territoriale sur le Nitaskinan soit un succès du point de vue nehirowisiw, il est nécessaire d'en arriver à une entente de cogestion qui soit assez flexible pour inclure la vision évolutive des traditions et des institutions qu'épousent les Nehirowisiwok. Ce qui ne peut être réalisé que si la gestion partagée du territoire est vue comme un exercice flexible de construction et de révision, en continu, des institutions et des savoirs environnementaux, au rythme des transformations sociales autochtones.
Tous droits réservés @ Recherches amérindiennes au Québec, 2014
Ce document est protégé par la loi sur le droit d'auteur. L’utilisation des services d’Érudit (y compris la reproduction) est assujettie à sa politique d'utilisation que vous pouvez consulter en ligne.

https://apropos.erudit.org/fr/usagers/politique-dutilisation/ 


\section{Nicolas Houde}

Département de science politique, Université du

Québec à Montréal
Ce n'est pas de dire " c'est mon territoire, c'est le mien! » Juste dire comme ça... le gars qui dit ça, " c'est mon territoire », il ne sait pas gérer son territoire

(ka nikaniwitc domicilié à Wemotaci).

D EPUIS PLUS DE TRENTE ANS, la Nation nehirowisiw $^{1}$ (Québec, Canada) est engagée dans un processus de négociation avec les gouvernements fédéral et provincial dans le but à la fois de clarifier les questions de titre sur les terres, d'accroître son accès aux ressources naturelles et de transformer le système de gouvernance territoriale sur le Nitaskinan, son territoire ancestral. Les Nehirowisiwok visent, entre autres, à ce que la gouvernance territoriale se fasse en accord avec leur vision du monde et en mobilisant leurs institutions traditionnelles de prise de décisions (CNA 2004, 2006). Conséquemment, l'un des principaux enjeux de cette recomposition des institutions de gouvernance est la revalorisation du rôle traditionnel et central du ka nikaniwitc, chef de territoire nehirowisiw, dans les décisions concernant le territoire.

Cette volonté de revaloriser le rôle traditionnel du ka nikaniwitc et de le repositionner dans la gouvernance contemporaine du Nitaskinan soulève deux importantes questions auxquelles cet article tente de répondre. D'une part, il s'agit de savoir comment les Nehirowisiwok entrevoient le rôle contemporain du ka nikaniwitc et, d'autre part, de comprendre comment les ententes de cogestion entre
l'État et les Premières Nations pourraient permettre à des institutions traditionnelles actualisées telles que le ka nikaniwitc de s'insérer dans les processus décisionnels concernant le territoire. Lapproche actuellement utilisée au Canada pour négocier des ententes de cogestion comporte des obstacles qui limitent les autochtones, dont les Nehirowisiwok, dans leur démarche d'actualisation de leurs institutions traditionnelles, ces limites faisant obstacle à la conclusion d'ententes de cogestion satisfaisantes d'un point de vue autochtone. Je démontre par ailleurs plus loin qu'une approche plus flexible de cogestion adaptative peut apporter quelques éléments de solution dans la négociation d'ententes entre les Premières Nations et l'État.

Comme le suggère la citation reproduite en épigraphe, la gestion du territoire implique davantage que de simplement réclamer un titre de propriété. Pour bien gérer, il faut connaître le territoire et développer les institutions appropriées. Cet article s'attarde donc au processus réflexif ayant présentement cours au sein de la nation nehirowisiw à propos de la place que doivent occuper ses institutions traditionnelles dans la gestion $\mathrm{du}$ territoire et des ressources du Nitaskinan. Pour ce faire, je m'appuie sur les résultats de deux ateliers tenus en 2009 à Wemotaci et à Manawan, ainsi que sur des données provenant d'une soixantaine d'entrevues réalisées dans les trois communautés 
nehirowisiwok et au cours desquelles les participants se sont penchés sur le rôle contemporain de l'institution traditionnelle du ka nikaniwitc.

\section{COGESTION DU TERRITOIRE \\ ET ASPIRATIONS AUTOCHTONES}

"Le colonialisme au Canada constitue un rapport social qui s'accomplit essentiellement au détriment des peuples autochtones » (Green 2004 : 11). Afin de reconfigurer ce rapport social, une solution privilégiée depuis les années 1970, d'un point de vue autochtone, est la négociation d'ententes globales telles que les traités ${ }^{2}$ ou d'ententes plus limitées de cogestion, des ressources naturelles notamment. Dans le cadre de cet article, la cogestion réfère au partage du pouvoir et des responsabilités de gestion entre le gouvernement et un groupe autochtone à travers une certaine intégration des systèmes de gestion étatiques et locaux (Notzke 1995: 187). Cette définition est assez large pour « couvrir des réalités fort différentes qui vont de la simple consultation [des Autochtones par le gouvernement] à une réelle participation » autochtone aux décisions en gestion des ressources (Rodon 2003 : 105). Pour les Nehirowisiwok, deux voies peuvent être empruntées afin d'en arriver à conclure une entente de cogestion du territoire et des ressources: celle du règlement de leurs revendications globales par le biais d'un traité (présentement en négociation) ou celle de la négociation d'une entente qui se conformerait aux exigences de la Cour suprême du Canada établies en matière de consultation et d'accommodement au fil des vingt-cinq dernières années.

Certains voient dans la cogestion un processus permettant, d'une part, l'affirmation d'un «pouvoir autochtone » porteur d'un renouveau institutionnel au sein de la fédération canadienne (Papillon 2006 : 480) et, d'autre part, une meilleure participation, dans la pratique, aux décisions concernant le territoire et les ressources; un tel processus pouvant, de façon pragmatique, améliorer la qualité de vie matérielle des autochtones concernés (voir Salée et Lévesque 2010) et les pratiques environnementales. D'autres y voient plutôt une façon pour l'État d'intégrer de façon définitive les nations autochtones à l'intérieur d'institutions imaginées par et pour la société majoritaire, et à l'intérieur desquelles est réservée une certaine place à l'accommodement des pratiques locales, cela dans le but de maintenir les sociétés autochtones à la marge, sans réel pouvoir d'interférer avec le développement d'une identité, d'une économie et d'institutions par et pour la société majoritaire (voir p. ex. James Tully [2002], cité par Gagnon 2011 : 136). Or, il ressort souvent des revendications autochtones une volonté d'aller au-delà d'un simple accommodement et de recomposer les structures de gestion partagée du territoire en réservant une place légitime aux processus politiques traditionnels autochtones, volonté pouvant être perçue comme une forme de résistance culturelle et politique (Poirier 2010). Cela démontre une volonté de négocier d'égal à égal, en valorisant, au sein d'un système de gestion partagée, autant les structures politiques autochtones comme structures vivantes et évolutives, que les institutions de l'État. Tel que montré par Bowie (2013), les autochtones du Canada visent à utiliser leurs institutions et leurs savoirs traditionnels afin de transformer la gestion et le développement des territoires, contribuant ainsi à améliorer leur propre condition et celle des territoires. Pour Anderson et ses collègues (2009, cité dans Bowie 2013), les Autochtones ont besoin, afin de connaître du succès dans la cogestion des territoires, de renforcer les identités communautaires (ce qui passe souvent par un ancrage dans les traditions des institutions de prise de décisions) et leur capacité à travailler collectivement dans le but d'atteindre des buts communs de structuration et d'autonomisation des communautés. La volonté d'améliorer les processus de cogestion, d'un point de vue autochtone, nécessite donc que l'on s'attarde, comme je le fais dans cet article, à la façon dont les autochtones réfléchissent et revitalisent leurs processus internes de prise de décisions.

Outre les Nehirowisiwok, plusieurs nations autochtones ont identifié le besoin de renforcer la capacité interne des institutions traditionnelles à générer une vision territoriale qui pourra servir de socle aux décisions prises par les comités de cogestion. Ainsi, on peut noter par exemple les efforts du Northern Tutchone Council du Yukon pour institutionnaliser, dans la gestion des pêches, des principes émanant de leurs lois traditionnelles guidant les relations entre humains et non-humains. Cela est réalisé en renforçant et en déployant une éducation ancrée dans les principes traditionnels de la nation à l'intention non seulement des membres de cette nation, mais également des autres acteurs, non autochtones, présents sur leur territoire traditionnel (Bowie 2013). On peut également noter les efforts de la nation haïda (ColombieBritannique), qui a organisé dans la première moitié des années 2000 des débats internes qui ont mené à la formulation d'un énoncé de vision pour la gestion des terres (le Haida Land Use Vision), énoncé qui a servi de base de référence pour la participation des Haïdas au processus provincial de planification stratégique du développement du territoire (Haida Nation et British Columbia 2009). Cet énoncé de vision a en effet servi de base en 2007 à un accord de planification stratégique négocié avec le gouvernement de la Colombie-Britannique qui, en plus d'être ancré dans une vision et des valeurs haïdas du territoire, a réduit les coupes forestières et créé un réseau d'aires protégées sur leur territoire ancestral (Bowie 2013). C'est donc en mobilisant et en renforçant la capacité à travailler en commun à l'interne, en renforçant une identité communautaire et en déployant un énoncé de vision territoriale ancré dans leurs valeurs traditionnelles que les Haïdas ont pu changer les pratiques forestières sur leur territoire.

La volonté de placer des institutions traditionnelles autochtones vivantes et évolutives au coeur des ententes de cogestion se butte souvent à certaines difficultés qui trouvent leur source dans le format actuel de plusieurs ententes négociées au Canada. Dans le cadre du présent article, deux de ces difficultés sont d'un intérêt particulier. Premièrement, de nombreux observateurs soulignent la 
difficulté de concilier, au sein d'un même processus décisionnel, l'utilisation des savoirs traditionnels autochtones et des savoirs identifiés comme scientifiques (p. ex. McGregor 2000; Nadasdy 2003; Rodon 2003; Bowie 2013). Les ententes de cogestion réservent bel et bien une place à l'utilisation des savoirs traditionnels dans la prise de décisions en gestion du territoire. On pourrait donc croire que deux types de savoirs, construits sur des bases épistémologiques et ontologiques différentes, pourraient apporter une bonne complémentarité dans la construction d'un savoir global à propos des territoires et des ressources à gérer. Or, l'engagement à mobiliser les savoirs traditionnels autochtones dans les ententes ne s'étend généralement pas à un engagement à questionner les valeurs fondamentales qui se situent au cœur de la gestion étatique des ressources (Nadasdy 2003; Houde 2007; Bowie 2013). Dans la pratique de la cogestion et tel que documenté par exemple par Nadasdy (2003), les participants non autochtones aux processus de cogestion acquiescent relativement aisément à l'intégration de données environnementales provenant des savoirs traditionnels qui sont compatibles avec les bases de données développées et contrôlées par les experts non autochtones, tout en ignorant les dimensions spirituelles et cosmologiques des savoirs traditionnels, considérés par les experts non autochtones comme étant incompatibles et non pertinents à la gestion contemporaine de l'environnement. Conséquemment, afin d'acquérir de l'influence dans un processus biaisé à la faveur des expertises non autochtones considérées comme plus crédibles que celles de chasseurs ou d'aînés autochtones, les participants autochtones en viennent à se conformer au langage, aux pratiques et aux institutions technicoscientifiques du pouvoir (Nadasdy 1999). Cette dynamique contribue ainsi à consolider des bases de données créées et contrôlées en dehors des communautés locales et collabore à des priorités de recherche et de gestion territoriale formulées par des bureaucrates, des conseillers politiques et des experts non autochtones.

La difficulté de l'intégration des savoirs est donc en partie un problème politique. C'est une question de pouvoir qui n'est pas complètement transféré vers les autochtones et surtout vers les institutions locales, traditionnelles, auxquelles ils s'identifient et qui sont organisées en fonction d'une base cosmologique qui leur est propre et qu'il est difficile de faire valoir dans un processus de cogestion. Sans un véritable pouvoir de développer et de mettre en application leurs propres systèmes de valeurs, les institutions traditionnelles peuvent difficilement se valoriser, se mobiliser pour proposer de nouvelles solutions qui pourraient être testées en gestion de l'environnement, questionner et critiquer les approches de gestion du territoire et contribuer à leur transformation. Il devient également difficile, dans ce contexte, d'insuffler l'énergie nécessaire à la valorisation des institutions traditionnelles, à leur déploiement et à leur développement en tant qu'entités autonomes capables d'évoluer en fonction d'un contexte environnemental, politique et social changeant. Dans le contexte actuel, le pouvoir est concentré dans les mains des bureaucrates plutôt que dans les mains des institutions traditionnelles, ce qui fait que systématiquement, au Canada, les ententes de cogestion débouchent sur des processus calqués sur les bureaucraties non autochtones parlant un langage techno-scientifique (Peters 1999; Nadasdy 2003; White 2006).

Le deuxième problème lié à la volonté de placer des institutions traditionnelles autochtones vivantes et évolutives au cour de la gestion territoriale touche de façon importante les processus de cogestion découlant de la négociation des traités modernes au Canada, négociation à laquelle participent les Nehirowisiwok. Le problème découle de la difficulté d'adaptation des institutions de cogestion à un contexte social ou environnemental changeant. Ce manque de flexibilité découle du désir, de la part du Canada, d'en arriver à des ententes finales qui mettent un terme définitif aux revendications des groupes autochtones signataires. « Le gouvernement canadien est prêt à acheter la paix, mais il veut le faire une fois pour toutes, sans qu'il soit possible de rouvrir le dossier. » (Dupuis 2001 : 105) Le Canada vise par ces ententes à coucher sur papier l'ensemble des droits d'un groupe qui seront appliqués afin de ne pas avoir, dans le futur, à entrer à nouveau dans de longues négociations. Conséquemment, la possibilité d'adapter les ententes au contexte - social, économique et environnemental - changeant se trouve restreinte. Cette approche enlève aux générations autochtones à venir la possibilité d'utiliser le traité comme instrument de mise en ouvre de leur propre vision de la gestion et du développement du territoire si cette vision diffère de celle des signataires originaux du traité.

Dans ce contexte, lorsque les Cris de la Baie James, signataires d'un traité datant de 1975, voulurent adapter dans les années 1990 les pratiques forestières sur leur territoire afin de s'adapter à un contexte économique et social changeant (il n'y avait pratiquement pas de foresterie en territoire cri en 1975), ils durent recourir aux tribunaux afin de forcer la main du Québec, la Province étant peu désireuse de modifier les pratiques qui avaient été établies par le traité, même si celui-ci n'abordait que de façon marginale la question de la foresterie (Grand Conseil des Cris 1998). Il y avait, d'un point de vue cri, une nécessité de modifier les pratiques de cogestion afin de fournir à une nouvelle génération de Cris les outils leur permettant de déployer leur vision territoriale dans un contexte où la foresterie industrielle prenait une place beaucoup plus importante qu'en 1975, mais la fermeté de la Province et la rigidité des institutions de cogestion rendaient inévitable le recours aux tribunaux. Le contexte institutionnel de la cogestion fut finalement modifié en 2002 par l'entente qu'on appellera la Paix des Braves, mais l'épisode a démontré les limites d'adaptation des traités.

Les ententes de cogestion négociées à l'extérieur des cadres des revendications globales peuvent partiellement répondre à ce problème d'adaptation des institutions, mais elles ne sont pas elles-mêmes exemptes de tout problème. Ainsi, en faisant pression sur les Autochtones pour rendre 
l'exercice des droits autochtones (à travers les ententes de cogestion) compatible avec les institutions canadiennes contemporaines, la cogestion pourrait limiter le déploiement de la spécificité culturelle des institutions de prise de décisions autochtones. Bernard Cleary, ancien négociateur des Nehirowisiwok dans le dossier de leurs revendications globales, décriait cette pression dans les années 1990 :

Nos droits ancestraux [...] sont équivalents à des droits de souveraineté. Il est évident que nous n'accepterons jamais que ces droits soient limités à la notion étroite de droits résiduels de chasse, de pêche et de piégeage que veut nous appliquer sournoisement le gouvernement du Québec. (Cleary 1993 : 52)

Les limites identifiées dans les derniers paragraphes font donc obstacle à l'objectif autochtone d'adapter les institutions traditionnelles, d'expérimenter avec elles, même, afin de trouver comment, dans le monde contemporain, ces institutions porteuses d'une culture et d'un savoir territorial distincts peuvent s'insérer à l'intérieur de processus de cogestion pouvant suivre le rythme des transformations sociales autochtones. De surcroît, du point de vue de la gestion de l'environnement et des ressources, les obstacles érigés face aux savoirs écologiques autochtones expliqués précédemment et la rigidité des approches de cogestion généralement négociées avec les Autochtones du Canada sont un anachronisme dans un monde de plus en plus caractérisé par la rapidité des transformations écologiques (Armitage et al. $2007: 1$ ).

Pour pallier les difficultés qu'ont les institutions de cogestion à s'adapter à des contextes environnementaux et sociaux qui changent parfois rapidement, un nombre croissant de chercheurs en gestion de l'environnement suggèrent maintenant d'emprunter une approche de cogestion adaptative de l'environnement (Folke et al. 2005; Armitage et al. 2007; Armitage et al. 2009; Berkes 2009). La cogestion adaptative est un processus flexible permettant aux partenaires de gestion de tester, réviser et améliorer par la pratique leur connaissance de l'environnement, leurs méthodes de gestion, ainsi que leurs processus décisionnels (Folke et al. 2002; Armitage et al. 2007). Lapprentissage et l'expérimentation active sont au cœur de ce processus qui permet une révision et une amélioration en continu des arrangements institutionnels, des méthodes de gestion et de politiques environnementales. En ce sens, les ententes de cogestion deviennent des occasions pour construire un nouveau savoir territorial et de nouvelles institutions (Berkes 2007). Cette approche se démarque des processus de cogestion actuellement négociés avec les Autochtones du Canada par l'institutionnalisation non pas des structures décisionnelles, mais de l'apprentissage et de l'innovation.

Ce que cet article suggère est que cette approche de cogestion adaptative peut apporter quelques améliorations aux ententes de cogestion négociées notamment dans le contexte nehirowisiw. Les prochaines sections de l'article vont montrer qu'une approche de cogestion plus flexible doit être envisagée afin d'inclure la vision évolutive des traditions et des institutions qu'épousent les Nehirowisiwok et de pouvoir tester dans la pratique, à l'intérieur d'un cadre flexible, comment peut se jouer le rôle contemporain du ka nikaniwitc.

\section{Nisawitatan Kitaskino}

Au cours de l'hiver 2009, des gens des communautés de Wemotaci et de Manawan se sont rencontrés pour discuter de la façon dont ils voyaient l'avenir de la gouvernance territoriale du Nitaskinan. Une cinquantaine de personnes, dont plusieurs chefs de territoire, ont participé à des ateliers afin de trouver ensemble des solutions aux problèmes qu'ils vivent en territoire. De plus, une soixantaine d'entrevues semi-dirigées ont été menées dans les trois communautés nehirowisiwok, soit Manawan, Opitciwan et Wemotaci. Quatorze femmes et 46 hommes provenant de différents groupes sociaux de la nation ont participé à ces entrevues, lesquelles se sont déroulées tantôt en français, tantôt en langue nehirowisiw.

Ces ateliers et ces entrevues se sont déroulés dans le cadre du projet Nisawitatan Kitaskino, un projet de recherche collaborative piloté et financé en grande partie par le Conseil de la Nation atikamekw (CNA). L'expression « Nisawitatan Kitaskino » évoque en langue nehirowisiw l'idée de la planification stratégique du territoire. Le projet avait pour but d'amorcer une réflexion qui devait mener, à terme, à la mise en place de nouvelles structures pour une « meilleure vie » en territoire pour les familles nehirowisiwok. Le projet s'est réalisé dans la perspective d'identifier des lignes directrices pour la gestion territoriale permettant à la nation d'aménager et développer le territoire selon sa vision propre et en respect de ses droits ancestraux. Je m'attarde dans les prochaines sections au rôle contemporain des chefs de territoire tel que défini par les participants aux ateliers et aux entrevues.

\section{LE CHEF DE TERRITOIRE, HIER ET AUJOURD'HUI}

Avant l'établissement des colons au Nitaskinan, les Nehirowisiwok formaient une société de chasseurs-cueilleurs autonomes (Poirier 2001; Wyatt 2004). Ce mode de subsistance ou d'économie mixte (en partie fondée sur la commercialisation de fourrures) - qui a persisté jusque dans les années 1950, après l'effondrement du commerce des fourrures, alors que les Nehirowisiwok commencèrent à travailler pour les compagnies forestières de plus en plus présentes sur leur territoire (Poirier 2001; Gélinas 2003; Morissette 2007) - signifiait que chaque famille étendue dépendait d'un territoire familial pour sa subsistance. Les familles pouvaient se rencontrer à des sites de rassemblement estivaux pour, entre autres, discuter de l'utilisation à venir des ressources du territoire et ensuite retraiter vers leurs territoires familiaux pour la chasse et le piégeage d'hiver. Laffiliation des familles à des territoires particuliers pouvait demeurer constante dans le temps, mais des mécanismes favorisant temporairement l'hospitalité et les échanges de territoire permettaient d'aider des familles qui se trouvaient dans le besoin si les ressources en nourriture n'étaient pas disponibles de façon uniforme, par suite, par exemple, de feux de forêt. C'est ce qui fait dire à Stephen 
Wyatt (2004: 91) que les territoires familiaux devraient donc probablement être compris comme des subdivisions flexibles du Nitaskinan se modifiant afin de refléter les besoins changeants de la société, et non comme des propriétés privées délimitées de façon rigide.

$\mathrm{Au}$ centre de ce système des territoires familiaux, des atoske askiwa, se trouve un chasseur accompli, le $k a$ nikaniwitc, le chef de territoire. Celui-ci a la responsabilité, entre autres, de répartir l'utilisation du territoire et des ressources entre les membres de sa famille, selon les saisons et les lieux, comme l'explique un chef de territoire d'Opitciwan :

Il gère la fréquentation, il gère la cueillette du gibier. Si à une place [il y a] du lièvre, on va aller dans ce coin-là, mais s'il voit que c'est en diminution, il va choisir [de] laisser reposer ce territoire là pendant un an ou deux, le temps que ça revienne. On va [alors] camper à une autre place.

Linstitution du ka nikaniwitc existe encore aujourd'hui, et les familles utilisent toujours le système des territoires familiaux pour déterminer l'occupation et l'espace où se pratiquent la chasse et les autres activités à caractère territorial. De plus, comme l'explique un jeune chasseur de Manawan, tout individu désirant occuper un territoire, y chasser ou y pêcher devrait recevoir, encore aujourd'hui, l'aval du ka nikaniwitc concerné, le responsable du territoire concerné :

Quand tu veux aller à la chasse dans un territoire [de la famille] Flamand, par exemple, il faut que tu ailles voir le chef de ce territoire-là. Tu lui demandes la permission pour aller là-bas. C'est ça, un chef de territoire. C'est lui qui refuse ou qui approuve tout ce qui se passe, là, sur son territoire.

Cette responsabilité de chef de territoire est habituellement transmise de père en fils (Poirier 2001), mais des femmes occupent de temps à autre ce rôle. L'hérédité n'est cependant pas le seul facteur déterminant dans la sélection d'un nouveau ka nikaniwitc. Un (ou une) chef de territoire peut être sélectionné(e) à cause de ses qualités de leadership, de son intérêt pour les questions territoriales, ou pour l'étendue de ses connaissances relatives au territoire. Il ou elle doit être « capable de gérer son territoire » (informateur d'Opitciwan). Un ka nikaniwitc connaît le territoire parce qu'il (ou elle) « est souvent là » (chef de territoire de Wemotaci) et peut donc «surveiller [avec l'aide de la famille] le territoire » (jeune femme de Manawan). Le ka nikaniwitc est aussi un ou une porte-parole pour la famille quand vient le temps de discuter des questions territoriales avec les autres familles ou avec d'autres acteurs territoriaux comme, par exemple, les compagnies forestières opérant sur leur territoire. Finalement, le ka nikaniwitc peut agir comme médiateur lorsque des tensions - entre familles ou à l'intérieur de sa propre famille - se font sentir, comme le souligne cet autre chef de territoire d'Opitciwan : « En tant que leader sur le terrain, c'est toi [le chef de territoire] qui dois gérer un peu les pour et les contre, les chicanes... Parce qu'il y a des chicanes [dans les familles], veut, veut pas. » Maintenant dans la trentaine, un employé des services de santé de Manawan qui se rend souvent pêcher sur le territoire de sa famille se souvient du temps où, jeune homme, il ne respectait pas toujours les limites de son territoire familial, forçant ainsi un ka nikaniwitc d'une autre famille à intervenir auprès de son père afin de faire respecter les règles relatives à la pêche :

On ne va jamais dans le territoire d'un autre. Comme moi, c'est arrivé deux ou trois fois [et] mon père [un aîné] me l'a dit : " II ne faut pas aller par là-bas, ce n'est pas notre territoire. » Parce que l'autre aîné est allé dire à mon père " J'ai vu ton fils là-bas... c'est notre territoire, ça. » Ce qui fait que ça a marché comme ça.

Aujourd'hui encore, donc, les membres des familles consultent leur ka nikaniwitc à propos de la pêche et de la chasse. Les chefs de territoire sont aussi sollicités, à travers un bureau de liaison existant au sein des conseils de bande, pour les consultations relatives à la planification du développement du territoire, notamment la planification forestière (voir Wyatt 2004 pour un compte rendu détaillé du processus de consultations pour la communauté de Wemotaci). Les ka nikaniwitcik ont ces responsabilités au sein des processus consultatifs parce qu'ils (ou elles) sont perçu(e)s comme étant les gardien(ne)s des territoires et d'une tradition; ils (elles) sont dès lors responsables de la transmission aux jeunes générations de cette tradition et des savoirs relatifs au territoire. Il revient donc aux chefs de territoire "d'assurer la survie de la culture », expliqua un participant de Wemotaci.

Par le maintien de l'institution des chefs de territoire et la participation de ceux-ci aux processus consultatifs, les Nehirowisiwok tentent donc à la fois de maintenir leur différence culturelle et de répondre aux exigences posées sur eux par l'État, notamment à travers la gestion bureaucratique des forêts du Nitaskinan, tel que souligné notamment par Sylvie Poirier (2010) et Stephen Wyatt (2004). La prochaine section vise à montrer comment les Nehirowisiwok conçoivent aujourd'hui l'expansion du rôle du ka nikaniwitc: allant d'un rôle de "consulté » sur les questions territoriales à un rôle de force motrice dans le développement d'une vision territoriale autochtone mise en œuvre par le biais d'une gestion territoriale renouvelée au sein de laquelle les familles nehirowisiwok joueraient un rôle actif.

\section{Demain : Autonomie Centrée sur des INSTITUTIONS TRADITIONNELLES CONTEMPORAINES}

$\mathrm{Au}$ cours des dernières décennies, les institutions traditionnelles des Nehirowisiwok ont été malmenées par la colonisation graduelle du territoire et des structures politiques (l'imposition du système des conseils de bande a dépossédé les ka nikaniwitcik de leur pouvoir d'influencer la gestion du territoire au profit des membres élus des conseils), par le développement de la foresterie commerciale et de l'industrie de la pêche et de la chasse sportives, ainsi que par la fragilisation de la transmission orale des traditions, due notamment à la mise en place d'obstacles à l'occupation territoriale et à l'épisode des pensionnats (tel que mentionné, par exemple, par Lavoie 1999 et Poirier 2010). Cependant, comme nous le verrons dans 
cette section, c'est autour du noyau des institutions traditionnelles du ka nikaniwitc et des territoires familiaux que les Nehirowisiwok d'aujourd'hui veulent construire leur autonomie, leur capacité à développer une économie basée sur une occupation territoriale cohérente avec leur vision territoriale et leurs objectifs de développement social. Chez les Nehirowisiwok, la définition d'autonomie va au-delà de ses aspects politiques et économiques. C'est aussi une question d'identité pour une société qui valorise la capacité individuelle d'être autonome en territoire (Poirier et Niquay 1999). Le terme «nehirowisiw» luimême, ethnonyme associé officiellement aux Atikamekw depuis 2006 (Jérôme 2008), fait d'ailleurs référence à une identité issue du territoire. C'est une identité, comme l'expliquent la Société d'histoire atikamekw et Laurent Jérôme (2009 : 24), qui dépend du territoire et « de la manière de s'adapter à celui-ci ». Un Nehirowisiw, c'est « celui qui vient du bois [...]. C'est un être indépendant de tous» (ibid.). C'est une personne capable de subvenir à ses besoins et à son bien-être, une personne qui est « en harmonie avec lui, avec les autres et avec son environnement» (Gilles Ottawa 2001, cité dans Société d'histoire atikamekw et Jérôme, 2009). Conséquemment, « du point de vue des aînés, c'est la responsabilité et l'engagement envers le territoire qui permettent à chacun de devenir un nehorisiw, soit celui qui a trouvé sa voie, son équilibre, son autonomie » (Poirier et Niquay 1999 : 21). Un Atikamekw, donc - ou un Nehirowisiw - est autonome en territoire, est capable de vivre en territoire, et l'autonomie dans la gestion du territoire devient une question d'identité, liée à la fierté d'être à la fois autonome et Atikamekw, comme le rappelle un enseignant à l'école secondaire de Wemotaci :

Un Atikamekw, ça a un rôle de gestion. Et il faut se réapproprier cette responsabilité-là. II faut se réapproprier la gestion de notre territoire. II faut se réapproprier l'engagement qu'on a toujours eu vis-à-vis le territoire.

Dans un contexte de négociation d'un traité, cet engagement envers le territoire et cet engagement à être nehirowisiw, un être autonome en territoire, se traduisent par des revendications ayant comme objectif de donner la capacité à l'institution traditionnelle des ka nikaniwitcik à transformer, par exemple, les règlements appliqués à la pratique de la foresterie afin de les rendre compatibles avec une éthique nehirowisiw du territoire. Cet engagement se traduirait également par la capacité de créer un cadre légal favorisant un accès et une occupation plus faciles du territoire par les Nehirowisiwok, ce qui permettrait, tel que souvent mentionné dans les ateliers et les entrevues, de développer une économie nehirowisiw en territoire, par le biais d'entreprises familiales dans les domaines, par exemple, du tourisme ou de la commercialisation de produits forestiers non ligneux (champignons, thé, etc.). Cette approche, passant par l'autonomie des familles, est donc une façon de développer une approche contemporaine de vivre en territoire compatible avec les valeurs nehirowisiwok d'autonomie, redonnant par ailleurs un rôle de premier plan au ka nikaniwitc dans la définition de l'orientation à donner aux activités se déroulant dans le territoire. Il n'est donc pas surprenant, étant donné la vision nehirowisiw de l'autonomie, autonomie ancrée dans la culture et la tradition, que l'existence du $k a$ nikaniwitc, et sa revitalisation en tant qu'institution légitime de prise de décisions, semble être une condition préalable sine qua non à la transformation de la gestion territoriale au Nitaskinan: «Si ça c'est brisé [l'institution des chefs de territoire], c'est une révolution qui va arriver » (un employé du Conseil de bande de Wemotaci).

La figure ci-contre synthétise la vision qu'ont les participants au projet Nisawitatan Kitaskino du rôle du $k a$ nikaniwitc dans le monde contemporain. Ce n'est pas une représentation du fonctionnement actuel de la gouvernance territoriale au Nitaskinan, mais plutôt une vision d'un processus de prise de décisions idéal. Dans ce schéma, le chef de territoire est positionné comme un catalyseur à travers qui circule l'information territoriale. Cette information est organisée par le ka nikaniwitc pour produire des décisions qui, une fois implantées, auraient des impacts à différentes échelles, du territoire familial au territoire ancestral national et au-delà.

La première responsabilité d'un chef de territoire est de connaître son territoire et de savoir ce qui s'y passe : "un chef de territoire, il connaît son territoire » (un chef de territoire de Manawan). Être chef de territoire, c'est aussi « être capable de donner [...] les bonnes informations au reste de son clan» (enseignant à l'école de Manawan). Linformation dont il est question ici est l'information qui sera nécessaire pour prendre des décisions éclairées quant à l'utilisation des ressources, mais également celle qui est nécessaire à la définition d'une prise de position de la famille dans le but, par exemple, de négocier avec les compagnies forestières concernant l'exploitation des ressources sur le territoire familial. Linformation utilisée par le $k a$ nikaniwitc et sa famille provient, comme le montre la figure ci-contre, des inventaires générés par l'occupation active du territoire, mais également des données générées par les autres acteurs présents sur le territoire, dont les compagnies forestières ou les agents du gouvernement. Cette information, une fois assemblée par le ka nikaniwitc (voir fig. : partie inférieure), est organisée de façon à pouvoir être utilisée pour faciliter le consensus au sein de la famille quant aux décisions à prendre (voir fig. : cercle « Famille»). Sont également considérées dans la réflexion de la famille les ententes conclues dans le passé avec les familles voisines, ainsi que celles devant être négociées ou renégociées dans le but d'éviter les conflits au sein de la communauté (cercle «Communauté »). Une fois identifié un consensus sur la voie à emprunter, le ka nikaniwitc s'assure de sa mise en œuvre. Les décisions émergeant ainsi de ce processus de concertation des points de vue familiaux et d'agrégation de l'information disponible devraient avoir un impact sur le fonctionnement du territoire familial, ainsi que sur les activités d'aménagement proposées par des acteurs extérieurs à la famille, sur les lois et règlements pouvant affecter le territoire, le développement du territoire, ou sur la 
santé globale des écosystèmes à différentes échelles (fig. : partie du haut).

Le point focal de ce système est donc le ka nikaniwitc et sa famille. Le $k a$ nikaniwitc enseigne et est attentif aux besoins de sa famille, comme le rapporte ce chasseur de Wemotaci, fils d'un chef de territoire:

Le chef de territoire, c'est mon père. La
première chose que je vois, c'est mon
père en premier. Mon père [...] nous a
transmis bien des affaires, des connais-
sances à lui. Moi, je pense que c'est son
rôle de communiquer son expérience de
vie, tout ce qu'il a appris, tout ce qu'il a
connu, tant au point de vue personnel
qu'au point de vue environnement... les
changements qu'il y a eu, tout ça.

Le chef de territoire tente de créer le consensus parmi les membres de sa famille, sur la base de l'information disponible et de la contribution de chacun :

[Le chef de territoire] consulte les autres membres de la famille puis il fait ses déductions [et] s'en va avec ça. La plupart du temps, il suit nos recommandations [des autres membres de la famille]. (Homme d'Opitciwan fréquentant régulièrement le territoire)

De par sa position de leader et de faiseur de consensus, le chef de territoire est donc appelé à jouer le rôle de représentant familial auprès des autres acteurs du territoire:

[Un chef de territoire], c'est une personne qui peut faire toutes les relations avec les membres de la famille, que ce soit au niveau social ou au niveau politique ou même économique. II faut que cette personne-là puisse être le porte-parole du groupe. [Les décisions] vont être [prises] par consensus, mais ça va être plus un porte-parole. (Fils d'un chef de territoire de Wemotaci)

Conséquemment, comme cette jeune femme de Manawan l'explique, le chef de territoire n'est pas seul responsable du territoire. Les décisions doivent se baser sur un consensus établi entre les membres :

Il ne doit être pas le seul à être responsable [...]. Nous [les autres membres de la famille] sommes là pour surveiller le territoire et, si on rencontre un problème sur le territoire, c'est lui qui doit parler, mais pas seulement avec sa façon de penser. II doit consulter ses frères et sœurs, ses neveux, sa mère... II doit demander leur avis [concernant ce] qui doit être fait s'il y a du développement en territoire, des négociations territoriales ou bien des coupes forestières.

À l'échelle de la communauté (fig. : cercle « Communauté »), le chef de territoire amène la famille à discuter des décisions territoriales qui pourraient, si mises en œuvre, avoir un impact au-delà de la famille concernée. Conséquemment, le ka nikaniwitc, dans la planification territoriale, doit créer des ententes, des alliances, avec les familles voisines. Les chefs de territoire sont donc appelés à discuter des questions territoriales ensemble lorsqu'elles concernent plusieurs familles et lorsqu'une direction générale est nécessaire à la vision du développement territorial communautaire. Conséquemment, presque tous les participants aux ateliers supportent l'idée de créer, dans un avenir rapproché, une organisation facilitant les discussions entre familles, dans le but de créer une vision territoriale commune et d'affermir la posture de la nation atikamekw dans ses négociations avec les acteurs gouvernementaux ou l'industrie forestière. Une telle organisation ayant pour but d'accroître l'influence des Nehirowisiwok sur le territoire ancestral est d'ailleurs en cours de création sous la forme d'un « conseil de territoire » (Poirier 2010).

Une telle organisation contribuerait à permettre aux $k a$ nikaniwitcik d'assumer à l'échelle du territoire (fig. : cercle « Territoire ») les responsabilités qui leur incombent, telles qu'identifiées par les participants aux ateliers, c'est-à-dire faire un suivi environnemental qui contribuerait à la définition de nouvelles règles de gestion forestière pour l'ensemble du territoire. Sur le territoire, l'impact de l'action du ka nikaniwitc se fait sentir à travers l'implantation 
de codes de pratiques basés sur un code d'éthique nehirowisiw de vie en territoire. Alors que ce code est mis à jour par l'expérience venant de la pratique, il est en outre amélioré avec l'expérience et sert de canevas à la planification forestière et à la cohabitation avec les autres acteurs du territoire. C'est donc de cette façon, par la contribution d'un code de pratiques cohérent avec la cosmologie nehirowisiw, que se fait, dans un monde idéal, le lien avec le "monde extérieur » (le cercle se trouvant aux confins de la figure).

Ce dernier cercle illustre le lien entre la tradition des Nehirowisiwok et le monde non autochtone. Il démontre une volonté de contribuer et d'utiliser les sources extérieures de savoir environnemental afin d'enrichir le savoir écologique traditionnel nehirowisiw. Il démontre également une volonté d'influencer les décisions relatives au territoire pour que celles-ci soient cohérentes avec la vision du monde nehirowisiw articulée par le ka nikaniwitc et sa famille. Le résultat est une intégration de l'information provenant de différentes sources à l'intérieur d'un cadre de référence nehirowisiw dont les caractéristiques et les balises sont définies par la famille et le ka nikaniwitc. Cette intégration sert ensuite à définir une orientation à donner aux politiques et actions de gestion territoriale, orientation que le ka nikaniwitc devra négocier, défendre, auprès des autres acteurs du territoire, des compagnies forestières et des gouvernements non nehirowisiw, comme l'expliquent ces participants aux entrevues :

Le [...] chef de territoire, [serait] un de nos représentants. Un chef de territoire est là pour [être] un porte-parole, il est là pour amener au-devant des choses qu'on envisage de faire. [II serait représentant auprès] du gouvernement autochtone [... ], si jamais ça existe, un gouvernement autochtone [et] qu'il soit représentatif sur les deux côtés, auprès des non-autochtones et des autochtones. (un jeune homme de Manawan)

[Le chef de territoire est] un rassembleur, il donne des informations, il va négocier en notre nom. C'est un leader, qui va être un porte-parole qui va défendre notre idée. (une employée de l'école primaire d'Opitciwan)

Le ka nikaniwitc n'est donc pas seulement un détenteur de savoir traditionnel, un enseignant et un médiateur, mais également un négociateur qui intervient dans les discussions avec les représentants des différents gouvernements, nehirowisiw, québécois ou canadien.

\section{TRADITION ET CONTEMPORANÉITÉ}

On constate à la lecture des résultats des entrevues et des ateliers que les thèmes identifiés comme faisant partie des responsabilités incombant à un chef de territoire ne se limitent pas uniquement à ce qu'on identifie habituellement comme étant des activités traditionnelles. Mark Nuttall (1998) nous rappelle que les non-autochtones ont encore tendance aujourd'hui à entretenir une image des activités et des savoirs traditionnels qui est celle d'un corpus cristallisé dans le passé, n'évoluant pas avec le temps. Or, les participants à la recherche soulignaient tous la nécessité pour le ka nikaniwitc de devenir plus engagé (qu'à l'heure actuelle) dans les décisions de développement économique, dans l'utilisation de nouvelles technologies afin d'améliorer le développement d'inventaires des ressources dans les territoires familiaux et dans l'atteinte de consensus sur la base des besoins d'aujourd'hui. Cela dévoile une volonté nehirowisiw d'être partie prenante dans la planification et le développement économique du Nitaskinan par l'entremise d'une concertation entre $k a$ nikaniwitcik, comme le mentionne ce chef de territoire d'Opitciwan :

Au niveau du développement économique territorial, on devrait voir ensemble [les ka nikaniwitcik] comment on pourrait faire, pour voir dans quelle mesure [on pourrait] aller chercher le développement économique.

Évidemment, réfléchir au renouvellement d'une institution politique traditionnelle telle que le $k a$ nikaniwitcik ou le conseil de territoire, nécessite de repenser le rôle du conseil de bande et sa relation avec les institutions traditionnelles. La difficulté est donc de repenser le rôle et le fonctionnement de chacun, même s'il est encore tôt, pour les Nehirowisiwok, d'élaborer des scénarios précis d'articulation entre les deux entités :

C'est là qu'est la confusion, ce que je vois comme confusion. II y a deux entités [...] et c'est ce qui se confronte en ce moment. II y a toute la facette culturelle, traditionnelle, qui est [celle des] chefs territoriaux, de territoire, les chefs de territoire familial versus les membres élus du conseil de bande. (fils de chef de territoire de Wemotaci)

Morissette (2007) souligne qu'effectivement il existe en ce moment au sein des communautés nehirowisiwok deux espaces politiques au niveau local : un formel, imposé par l'État colonial, et un autre, traditionnel, composé de ka nikaniwitcik, d'aîné(e)s, de gardiens de la tradition et fonctionnant en arrière-plan, sollicité occasionnellement à propos de certaines décisions importantes. Ce que les résultats des ateliers et des entrevues démontrent, résumés par le participant cité ci-haut, est qu'il existe une volonté de recomposer la gouvernance interne de la nation afin de créer un espace formel pour ces processus politiques prenant leur source dans la tradition nehirowisiw et pour « autochtoniser », pour reprendre l'expression de Green (2004), les processus décisionnels existants.

Les résultats du projet Nisawitatan Kitaskino montrent donc que les Nehirowisiwok désirent mettre sur pied un système de gouvernance auquel ils s'identifient, ancré dans la tradition, centré sur le chef de territoire et la famille, mais en même temps tourné vers l'avenir, évolutif et différent de sa forme originale. Dans le système proposé, le chef de territoire se positionne comme un catalyseur par lequel passe et s'organise l'information concernant le territoire afin de produire des décisions compatibles avec les valeurs nehirowisiwok et ayant des effets à plusieurs échelles (territoires familiaux, Nitaskinan, régions administratives du Québec). Selon ce modèle, les décisions territoriales concernées ne se limitent pas à ce qui est perçu généralement comme faisant partie du domaine des activités traditionnelles (ex. la chasse et la pêche), mais touchent également tous les aspects de la gouvernance territoriale, incluant la production de savoirs environnementaux. Pour que la transformation de la gouvernance territoriale au 
Nitaskinan soit un succès d'un point de vue nehirowisiw, il est donc nécessaire d'en arriver à une entente avec les gouvernements non autochtones qui soit assez flexible pour inclure la vision évolutive des traditions et des institutions qu'épousent les Nehirowisiwok. Ce qui ne peut être réalisé que si la gestion partagée du territoire est vue comme un exercice de recomposition et de construction des institutions et des savoirs territoriaux, tel que suggéré plus tôt dans le texte. Une telle approche voudrait notamment dire que le rôle des $k a$ nikaniwitcik ne serait pas cantonné à fournir aux compagnies forestières et aux biologistes des informations factuelles qui compléteraient des bases de données par des experts non autochtones, mais que leur rôle évoluerait en fonction d'une nouvelle réalité qui nécessite que le ka nikaniwitc prenne une part active dans la planification du développement économique en territoire, développement qui fait une place à des activités (comme le tourisme) qui ne sont pas perçues généralement par les non-autochtones comme étant des activités «traditionnelles ». Ce qui peut poser problème pour les non-autochtones lorsque vient le temps de négocier un rôle de cogestion pour les institutions traditionnelles. En effet, comme Nadasdy (2003) nous le rappelle, plusieurs non-autochtones d'Amérique du Nord voient dans les changements de pratiques ou de styles de vie des autochtones une preuve que leurs traditions et leurs identités disparaissent. Il est donc important, d'un point de vue autochtone, d'affirmer la légitimité des institutions traditionnelles, même si celles-ci se transforment.

\section{LES INSTITUTIONS TRADITIONNELLES DANS LA GESTION PARTAGÉE DU TERRITOIRE}

Deux difficultés associées à la négociation d'ententes entre l'État et les Autochtones ont été identifiées au début du texte, à savoir, premièrement, une difficulté à concilier, à l'intérieur d'un même processus décisionnel, les savoirs et les institutions identifiés comme traditionnels et ceux qui sont identifiés comme scientifiques et bureaucratiques; et deuxièmement, une difficulté d'adaptation des institutions de cogestion à un contexte social ou environnemental changeant. Nisawitatan Kitaskino nous indique que la négociation d'ententes de cogestion nécessite, afin de tenter de surmonter en partie ces deux difficultés, qu'on la conçoive comme une occasion de construire des institutions qui trouvent leur inspiration dans la tradition, permettant ainsi aux autochtones de s'identifier fortement à ces institutions. Un tel espace réservé à la création pourrait assurer que ces institutions soient tournées vers l'avenir, pouvant fonctionner dans le monde contemporain, alliant tradition et contemporanéité, et allant ainsi au-delà de simples reproductions des cultures gouvernementales de l'État canadien. Autrement dit, il faut en arriver à faire en sorte qu'à l'intérieur d'une entente de cogestion, les institutions autochtones, le ka nikaniwitc et le territoire familial, dans le cas des Nehirowisiwok, puissent évoluer de manière à ce que les Autochtones puissent se construire des institutions contemporaines de gouvernance qui fonctionnent, qui soient cohérentes avec la culture locale et qui puissent leur permettre à eux et à leurs gardiens de traditions d'adopter de nouvelles méthodes de construction du savoir, de nouvelles traditions de prise de décisions, selon les besoins et les valeurs de leurs communautés.

Pour y arriver, il est nécessaire que les ka nikaniwitcik soient habilités (empowered) à participer à la création des institutions de cogestion. Agrawal (1995) a déjà souligné à cet égard qu'afin de construire des processus décisionnels qui soient bénéfiques pour les populations locales, autochtones en l'occurrence, il faut concentrer les énergies à créer une plus grande autonomie habilitante pour ces populations afin qu'elles puissent décider de ce qui est approprié ou non pour la gouvernance de leurs territoires ancestraux et décider de ce qui est approprié pour leur communauté et leur culture en termes de développement territorial. Conséquemment, les Nehirowisiwok ont exprimé, à travers Nisawitatan Kitaskino, qu'ils croient nécessaire que les chefs de territoire prennent part aux décisions à propos de sujets allant au-delà des activités « traditionnelles » définies de façon étroite. Ils soutiennent plutôt que leur participation doit s'inscrire dans un processus de planification territoriale qui s'étend de l'élaboration des orientations stratégiques jusqu'au suivi des opérations sur le terrain (voir la figure). Cette vision nécessite donc de dépasser le simple modèle consultation/accommodement, actuellement prôné par les États canadien et québécois, et d'aller dans la direction d'une réelle contribution aux décisions territoriales et d'une mobilisation des ka nikaniwitcik dans la construction d'institutions de cogestion auxquelles participeraient des représentants tant du gouvernement que des Nehirowisiwok guidés ou mandatés par leurs institutions traditionnelles, ce qui permettrait à ceux-ci de renforcer leur culture politique distincte tout en conservant leur autonomie culturelle.

Une vision plus fluide, plus autonomiste, du droit des autochtones à participer aux décisions se démarque, comme mentionné précédemment, d'une perception courante chez les non-autochtones, à savoir celle d'une tradition autochtone figée dans le passé, inamovible. Pour le public non autochtone, la tradition, la marginalité et la continuité historique de la présence autochtone en territoire sont ce qui donne légitimité à une voix nehirowisiw dans la négociation d'arrangements de cogestion. Cette différence est ce qui peut servir de levier face à l'idéologie dominante de l'État. Sans ces traditions, inamovibles, sans cette différence, pourquoi négocier des arrangements spéciaux? Or, cette vision tend à ne pas tenir pour légitimes les processus de changements sociaux ayant cours dans les sociétés autochtones du Canada. Ces transformations sociales, la volonté croissante, par exemple, de développer une économie locale et entrepreneuriale qui va au-delà de la simple subsistance, doivent pourtant être prises en considération lorsqu'on tente de mettre en œuvre ce que les participants à Nisawitatan Kitaskino identifient comme une bonne gouvernance territoriale. Jérôme écrit à ce propos :

Les Premières Nations actuellement engagées dans des processus de revendication face aux États qui les englobent doivent amener la preuve que ce qui définit l'essence de "l'être autochtone » est fidèle à un passé ancestral pour que soit simplement reconnu le droit d'entamer des négociations à propos de leurs spécificités 
culturelle, identitaire et territoriale. Cette essence de «l'être autochtone " n'est pas perçue à travers ce que les autochtones sont aujourd'hui mais en regard de ce qu'ils font avec ce qu'ils sont. Par exemple, les " activités traditionnelles » sont réduites par les systèmes juridique et politique, canadiens notamment, à un sens strict et minimum « d'activités de subsistance » (Jérôme 2005 : 21).

Cette nécessité de se plier à une telle définition des traditions force les autochtones à entrer dans un cadre qui limite la créativité des processus de négociation et la possibilité d'inventer un système de cogestion du territoire flexible et évolutif qui réponde aux objectifs contemporains des sociétés autochtones en termes de développement social et économique. Les Nehirowisiwok, notamment, sont donc jusqu'à maintenant contraints, dans leurs négociations territoriales, de choisir entre traditions indépassables ou une intégration/assimilation à travers une participation à des processus « modernes » dont les paramètres sont largement définis par l'État. Cela limite, comme nous l'avons vu plus tôt dans le cas de la Convention de la Baie James et du Nord québécois signée en 1975, la possibilité de créer des ententes de cogestion adaptées au contexte évolutif des populations locales. À cet égard, en se référant à la Convention, Philip Awashish (2005), ancien négociateur cri, souligne que l'entente s'est avérée être un instrument peu flexible, qui n'avait pas évolué de pair avec les réalités et les besoins locaux. Selon lui, pour les gouvernements non autochtones, le but était (en plus de l'accès au territoire) de s'entendre une fois pour toutes sur les façons de faire et d'éteindre tout droit qui n'aurait pas été prévu au traité, ou toute évolution des pratiques qui n'aurait pas été prévue en 1975. Awashish ajoute qu'en conséquence, l'entente demeure une expression partielle et incomplète des droits inhérents des Eeyou (Cris) à se gouverner (Awashish 2005: 180). Ce qui rejoint les propos de Bernard Cleary (qui ont été reproduits plus tôt dans ce texte) ainsi que les propos rapportés par les participants à Nisawitatan Kitaskino, à savoir que les droits que les Autochtones du Canada essaient de traduire en traités et en ententes de cogestion ne sont pas que des droits à la pratique d'activités de subsistance, mais des droits d'autonomie plus larges qui leur permettraient, notamment, de redéployer et remodeler leurs institutions traditionnelles de prise de décisions.

Ladoption d'un processus de cogestion adaptative des ressources et du territoire pourrait permettre aux Nehirowisiwok d'expérimenter avec l'institution des $k a$ nikaniwitcik, qui a été marginalisée pendant longtemps, afin de savoir comment elle pourrait vraiment s'insérer dans le monde contemporain de la prise de décisions territoriales tout en laissant une porte ouverte à de futurs changements à la suite de l'expérience de la gestion du territoire en commun (État et Autochtones). C'est en se concentrant sur l'apprentissage et en laissant la porte ouverte aux modifications des institutions et des pratiques «traditionnelles» que pourront véritablement s'insérer dans la prise de décisions les ka nikaniwitcik, permettant ainsi le succès, d'un point de vue nehirowisiw, d'une entente de cogestion.

\section{Conclusion}

Les travaux effectués dans le cadre du projet Nisawitatan Kitaskino montrent que les Nehirowisiwok tentent, par leur réflexion sur la gouvernance territoriale, de mettre sur pied un système auquel ils s'identifient, ancré dans la tradition, centré sur le chef de territoire et la famille, mais en même temps tourné vers l'avenir, évolutif, différent de sa forme originale et pouvant s'adapter aux besoins des futures générations. Dans le système proposé, le chef de territoire se positionne comme un catalyseur par lequel passe et s'organise l'information concernant le territoire afin de produire des décisions compatibles avec les valeurs nehirowisiwok et ayant des effets à plusieurs échelles (territoires familiaux, Nitaskinan, régions administratives du Québec). Selon ce modèle, les décisions territoriales concernées ne se limitent pas à ce qui est perçu généralement comme faisant partie du domaine des activités traditionnelles (ex. la chasse et la pêche), mais concernent également tous les aspects de la gestion du territoire, incluant la production de savoirs environnementaux. À partir de ces résultats, il est possible d'affirmer que pour que la transformation de la gouvernance territoriale au Nitaskinan soit un succès d'un point de vue nehirowisiw, il est nécessaire d'en arriver à une entente avec les gouvernements non autochtones qui soit assez flexible pour inclure la vision évolutive des traditions et des institutions qu'épousent les Nehirowisiwok. Cela ne peut être réalisé que si la cogestion du territoire est vue comme un exercice flexible de construction et de révision en continu des institutions et des savoirs environnementaux, et non comme un cadre strict à l'intérieur duquel sont atrophiées des pratiques traditionnelles de subsistance cristallisées dans le passé. Une telle approche, adaptative, qui adopte une vision évolutive des traditions autochtones, pourrait par ailleurs permettre de surmonter quelques obstacles qui existent actuellement dans les processus canadiens de cogestion. Elle donnerait la possibilité aux partenaires des ententes de trouver comment, dans le monde contemporain, les institutions porteuses d'une culture et d'un savoir territorial auxquels les autochtones s'identifient pourront s'insérer à l'intérieur de processus de cogestion, tout en suivant le rythme des transformations sociales autochtones et en évitant que ces institutions autochtones ne se dissolvent dans celles de la société majoritaire.

\section{Notes}

1. J'utiliserai dans cet article le terme «Nehirowisiw» (plur. Nehirowisiwok) pour nommer ceux qui jusqu'à tout récemment étaient désignés par l'ethnonyme «Atikamekw», cela conformément à la volonté de la Nation nehirowisiw.

2. Après l'arrêt Calder de 1973, le gouvernement fédéral fut forcé d'adopter une politique sur les revendications territoriales dans le but de négocier des traités avec les nations autochtones qui historiquement, comme les Nehirowisiwok, n'avaient jamais cédé leurs droits territoriaux.

\section{Remerciements}

Je remercie les membres du Secrétariat au territoire du Conseil de la Nation atikamekw pour leur appui et leur contribution à toutes les étapes du projet de recherche qui a mené à cet article. 
Des remerciements vont également aux participants aux ateliers et aux entrevues. Le travail de terrain a été rendu possible grâce au soutien financier du Conseil de la Nation atikamekw, ainsi que de l'Université McGill et du FQRSC.

\section{Ouvrages cités}

AGRAWAL, Arun, 1995: «Dismantling the divide between indigenous and scientific knowledge ». Development and Change 26(3) : 413-439.

ARMITAGE, Derek, Fikret BERKES et Nancy DOUBLEDAY (dir.), 2007: Adaptive co-management: collaboration, learning, and multi-level governance. UBC Press, Toronto.

ARMITAGE, Derek, et al., 2009 : «Adaptive co-management for social-ecological complexity ». Frontiers in ecology and the environment 7(2): 95-102

AWASHISH, Philip, 2005 : «From board to nation governance: The evolution of Eeyou Tapay-Tah-Jeh-Souwin (Eeyou governance) in Eeyou Istchee », in Michael Murphy (dir.), Reconfiguring Aboriginal-state relations : 165-183. McGill-Queen's University Press, Montréal.

BERKES, Fikret, 2007 : «Adaptive Co-Management and Complexity: Exploring the Many Faces of Co-Management », in Derek Armitage et al. (dir.), Adaptive Co-Management: Collaboration, Learning, and Multi-Level Governance: 19-27. UBC Press, Toronto.

—, 2009 : «Evolution of co-management: Role of knowledge generation, bridging organizations and social learning ». Journal of Environmental Management 90(5) : 1692-1702.

BOWIE, Ryan, 2013: «Indigenous Self-Governance and the Deployment of Knowledge in Collaborative Environmental Management in Canada». Revue d'études canadiennes 47(1): 91-121.

CLEARY, Bernard, 1993 : «Le long et difficile portage d'une négociation territoriale ». Recherches amérindiennes au Québec 23(1) : 49-60.

CNA (Conseil de la Nation atikamekw), 2004 : Cahier de propositions présenté dans le cadre des travaux de la Commission d'étude chargée d'examiner la gestion des forêts du domaine de l'État. Conseil de la Nation atikamekw, La Tuque.

—, 2006 : Mémoire déposé au Bureau d'audiences publiques sur l'environnement dans le cadre du Programme décennal d'épandage de phytocides par voie aérienne en milieu forestier sur des terrains privés de Smurfit-Stone inc. sur le territoire de La Tuque et de la MRC Domainedu-Roy. Conseil de la Nation atikamekw, La Tuque.

DUPUIS, Renée, 2001 : Quel Canada pour les Autochtones? La fin de l'exclusion. Boréal, Montréal.

FOLKE, Carl, et al., 2002 : Resilience and sustainable development: Building adaptive capacity in a world of transformations. International Council for Science, ICSU Series on science for sustainable development, no 3.

FOLKE, Carl, Thomas HAHN, Per OLSSON, et Jon NORBERG, 2005 : "Adaptive governance of social-ecological systems ». Annual Review of Environment and Resources 30:441-73.

GAGNON, Alain G., 2011 : L’Âge des incertitudes : Essais sur le fédéralisme et la diversité nationale. Presses de l'Université Laval, Québec

GÉLINAS, Claude, 2003: Entre l'assommoir et le godendart: les Atikamekw et la conquête du Moyen-Nord québécois, 1870-1940. Septentrion, Sillery.

GRAND CONSEIL DES CRIS, 1998 : No agreement in forestry case: Crees and Quebec talks but no agreement signed to withdraw from court. <http://www.gcc.ca/archive/article.php?id=31>, (consulté le 10 février 2014).

GREEN, Joyce, 2004 : « Autodétermination, citoyenneté et fédéralisme: pour une lecture autochtone du palimpseste canadien ». Politique et société 23(1) : 9-32.

HAIDA NATION et BRITISH COLUMBIA, 2009: Kunst'aa Guu Kunst'aayah Reconciliation protocol. <http://www.haidanation.cal Pages/Splash/Documents/Rec_Pro.pdf>, (consulté le 16 mai 2013).
HOUDE, Nicolas, 2007 : «The six faces of traditional ecological knowledge: Challenges and opportunities for Canadian comanagement arrangements ». Ecology and Society 12(2): art. 34. <http://www.ecologyandsociety.org/vol12/iss2/art34/>

JÉRÔME, Laurent, 2005 : « Musique, tradition et parcours identitaire de jeunes Atikamekw : La pratique du tewehikan dans un processus de convocation culturelle ». Recherches amérindiennes au Québec 35(3): 19-30.

—, 2008 : «"Faire revivre l'Indien au cour de l'enfant" : rituels de la première fois chez les Atikamekw Nehirowisiwok». Recherches amérindiennes au Québec 38(2-3) : 45-54.

LAVOIE, Kathia, 1999: Savoir raconter ou l'art de transmettre: Territoire, transmission dynamique et relations intergénérationnelles chez les Wemotaci Iriniwok (Haute-Mauricie). Mémoire de maîtrise, départment d'anthropologie, Université Laval, Quebec.

McGREGOR, Deborah, 2000 : « The state of traditional ecological knowledge research in Canada: A critique of current theory and practice ", in R.F. Laliberte et al. (dir.), Expressions in Canadian Native Studies : 436-458. University of Saskatchewan Extension Press, Saskatoon.

MORISSETTE, Anny, 2007 : «Composer avec un système imposé : la tradition et le conseil de bande à Manawan ». Recherches amérindiennes au Québec 37(2-3) : 127-138.

NADASDY, Paul, 1999 : «The politics of TEK: Power and "integration" of knowledge ». Arctic Anthropology 36(1-2) : 1-18.

_, 2003 : Hunters and bureaucrats: Power, knowledge, and Aboriginalstate relations in the Southwest Yukon. UBC Press, Toronto.

NOTZKE, Claudia, 1995: «A new perspective in aboriginal natural resource management: co-management ». Geoforum 26(2) : 187-209.

NUTTALL, Mark, 1998 : Protecting the Arctic: Indigenous peoples and cultural survival. Harwood Academic, Amsterdam.

PAPILLON, Martin, 2006 : « Vers un fédéralisme postcolonial? La difficile redéfinition des rapports entre l'État canadien et les peuples autochtones », in Alain G. Gagnon (dir.), Le fédéralisme canadien contemporain : fondements, traditions, institutions : 461-485. Presses de l'Université de Montréal, Montréal.

PETERS, Evelyn J., 1999 : « Native people and the environmental regime in the James Bay and Northern Quebec Agreement ». Arctic 52(4) : 395-410.

POIRIER, Sylvie, 2001: "Territories, identity, and modernity among the Atikamekw (Haut-St-Maurice, Quebec) », in Colin H. Scott (dir.), Aboriginal autonomy and development in Northern Quebec and Labrador: 98-116. UBC Press, Toronto.

- 2010 : «Change, Resistance, Accommodation and Engagement in Indigenous Contexts: A Comparative (Canada-Australia) Perspective ». Anthropological Forum 20(1): 41-60.

POIRIER, Sylvie, et Jean-Marc NIQUAY, 1999 : Le droit coutumier atikamekw: Pistes de réflexion. Non publié, juillet.

RODON, Thierry, 2003 : En partenariat avec l'État : Les expériences de cogestion des Autochtones du Canada. Presses de l'Université Laval, Québec.

SALÉE, Daniel, et Carole LÉVESQUE, 2010 : « Representing aboriginal self-government and First Nations/State relations: Political agency and the management of the boreal forest in Eeyou Istchee ». Revue internationale d'études canadiennes 41 : 99-135.

SOCIÉTÉ D'HISTOIRE ATIKAMEKW et Laurent JÉRÔME, 2009 : «Nehirowisiw kitci atisokan: vers une réappropriation de l'histoire atikamekw ». Les Cahiers du CIÉRA 4 : 19-30.

WHITE, Graham, 2006: «Cultures in collision: Traditional knowledge and Euro-Canadian governance processes in Northern land-claim boards ». Arctic 59(4) : 401-414.

WYATT, Stephen, 2004 : Co-existence of Atikamekw and industrial forestry paradigms, occupation and management of forestlands in the St-Maurice river basin, Quebec. Thèse de doctorat, Faculté de foresterie, Université Laval, Québec. 